\title{
Sensorless Vector Control of Permanent Magnet Synchronous Machine Using High-Frequency Signal Injection
}

\author{
Gergely Szabó, Károly Veszprémi
}

Budapest University of Technology and Economics, Faculty of Electrical Engineering and Informatics, Department of Electric Power Engineering, Egry József u. 18, 1111 Budapest, Hungary

szabo.gergely@vet.bme.hu, veszpremi.karoly@vet.bme.hu

Abstract: The vector control theory of alternating current machines could provide high performance control during transient events, since these methods do not depend on the static equations of the selected machine, but on the space vector-based differential system of equations. These control methods have a very important common property; all of them require an angle, with which the system's input can be transformed into the common reference frame in which the space vector notation is construed. The sensorless control methods attempt to estimate the common coordinate system's angle, without using any information from the encoder, one of which is the high-frequency voltage injection method. This paper presents the mathematical model of the high-frequency synchronous voltage injection method on permanent magnet synchronous machines. The common coordinate system is estimated using a Phase-Locked-Loop (PLL). Based on the detailed mathematical model a new equivalent dynamic model for the PLL structure is proposed, with which the $P L L$ 's controller could be tuned, with the knowledge of the machine's parameters and injected voltage properties. Simulation results are provided for an off-the-shelf interior magnet synchronous machine.

Keywords: Vector Control; Permanent Magnet Synchronous Machine; Sensorless; HighFrequency Signal Injection

\section{Introduction}

The sensorless vector control methods try to eliminate the speed encoder from the controlled electric drives, but in most cases, the shaft angle encoder cannot be omitted, because of the safety level of the application. In such drives, a sensorless vector control method can be used as a backup algorithm, with which the drive system can be stopped without damaging the drive or the users. The sensorless term must be clarified depending on the machine to be controlled; in case of an induction machine, this term needs to be divided into at least two approaches 
because the shaft angular position and the common coordinate system's angle, which is used to be rotor flux vector's position, are not the same and depends on the state of the machine. The simple sensorless term can be used for an algorithm, with which the common coordinate system can be estimated without using angle feedback from the shaft, but in this case, the closed-loop speed control still depends on the encoder attached on the rotor. On the other hand, a speedsensorless method is capable to control the induction machine's angular speed too, without any encoder built in the system. In the case of a synchronous machine, the sensorless and speed-sensorless terms can be merged since the pole-flux vector's angle is in direct relation with the shaft's mechanical angle.

From the algorithm point of view, the available methods could be categorized into two sections. The first one attempts to estimate the machine's signals based on its mathematical models and the combination of several filtering methods and controllers. Model Reference Adaptive System (MRAS) approaches are one of the well-known methods of this category. Authors in [1] detail a vector-controlled solution for permanent magnet synchronous machines, which is based on MRAS method. The control structure was constructed using the classical cascade PI speed and current control loops, the common coordinate system, which is required for the pole flux vector oriented vector control, was estimated using the MRAS method. Since angle estimation incorporates the entire machine equations, the precise knowledge of the parameters is required for a stable control over a wide range of synchronous frequency and temperature. Authors in [2] give a detailed case study of the parameter deviations' effect in an MRAS-based system, whilst [3] introduces an adaptive approach to overcome the uncertainty of the required parameters during operation. The computational effort of this method must be handled carefully, for which [4] proposes a reduced-order observer and neural network solution in terms of rotor flux estimation and compensation.

This category also incorporates the well-known estimator structures; such as Kalman filtering method, which also uses the machine's model, combined with model of the system's and the measurements' disturbances. Authors in [5] propose an Extended Kalman Filter-based (EKF) method, which does not require the knowledge of the mechanical parameters nor the initial rotor position. Since these algorithms are sample-based their implementation on pulse width modulated voltage-source inverter's microcontroller is straightforward. A major issue in these approaches is the selection of the covariance matrix, which is the result of a trial-and-error tuning in most of the cases. To overcome the difficulties of choosing the covariance matrix, [6] details an algorithm to select the appropriate parameters.

Sensorless algorithms in the second category try to exploit the machine's magnetic properties, which are dependent on the rotor's position or the magnitude of the flux. 
A well-known method of this category is the INFORM, Indirect Flux detection by On-line Reactance Measurement [7], which tries to track the machine's impedance with which the actual rotor position can be estimated. This algorithm does not involve any test signals, it just calculates the machine's impedances based on the measured currents and voltages. Similar to the previous MRAS solutions, the addition of an Extended Kalman Filter to this system can provide a better estimation.

The high-frequency signal injection methods are also part of the second group, in which methods usually voltage signals are injected into the predefined points of the control structure. The latter one leads us two main approaches; the highfrequency stationary injection modifies the phase voltages of the machine by adding a symmetric voltage system to the motor's terminal voltages [8-9]. In the second approach, the test vectors are injected in the estimated common reference frame, hence these techniques are called synchronous injection methods. Authors in [10] give a comparative analysis of the two aforementioned solutions. Besides the point of the injections, the signal processing methods with which the common coordinate system can be estimated are different.

The stationary injections usually involve heterodyne filtering techniques, in which the measured high frequency stationary currents are transformed into several coordinates systems in which they are filtered to obtain the required angle information [11]. In the case of synchronous injection, the flux vector's position can be estimated with Phase-Locked-Loop (PLL), but [12] proposes a discrete algorithm-based solution, which enables higher bandwidth in the estimation and speed control.

Section 2 presents the detailed mathematical model of high-frequency synchronous injection method on a permanent magnet synchronous machine. This is followed by the description of the common coordinate system's estimation, which is performed using a PLL. Based on the mathematical description a new dynamic model is proposed for the PLL-based estimator structure, which is required for most of the controller structures and their proper tuning.

The control structure assumed to be a widely used cascade control loop, containing $d$-direction current control loop, a $q$-direction current control loop which setpoint is provided by the outer speed controller loop. With the help of the new dynamic model the PLL's and the cascade control loop's PI controllers be tuned with one of the published methods to achieve a stable control. In Section 3 simulation results are presented using an off-the-shelf interior permanent magnet synchronous motor's parameters. 


\section{High-Frequency Syncronous Voltage Injection}

\subsection{Mathematical Model of Permanent Magnet Synchronous Machine}

The sinusoidal field, generated by an ideal permanent magnet can be modeled with a constant amplitude pole flux vector, which is bound to the point, where the rotor magnets' spatial flux density distribution reaches its maximum value. The common coordinate system is fixed to this vector because its cross product with the stator current defines the magnitude of the motor's torque. Equations (1)-(4) show the system of equations, which models the machine in the $d-q$ frame, whilst Fig. 1 shows the assumptions of the dynamic model, including the space vector-based equivalent circuit. In Fig. 1(b) $\overline{\bar{L}}$ denotes the $2 \times 2$ diagonal stator inductance matrix, which contains the $d$ - and $q$-direction inductances.

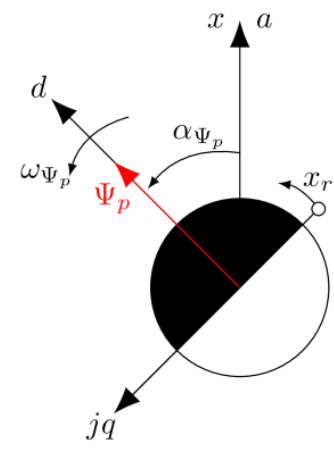

(a)

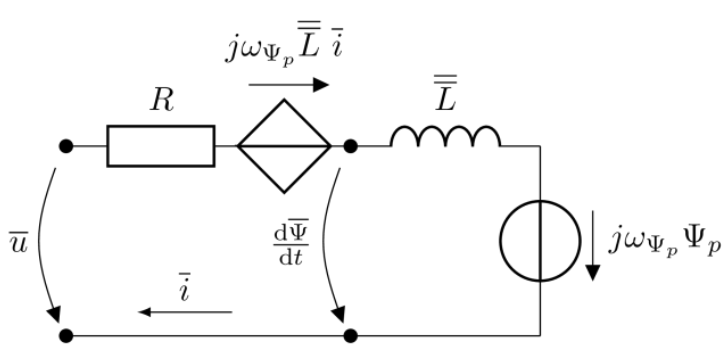

(b)

Figure 1

(a) Permanent magnet rotor and the definition of the common coordinate system's angle,

(b) Equivalent circuit of permanent magnet synchronous machine

$u_{d}=R i_{d}+L_{d} \frac{d i_{d}}{d t}-\omega_{\Psi_{p}} L_{q} i_{q}$,

$u_{q}=R i_{q}+L_{q} \frac{d i_{q}}{d t}+\omega_{\Psi_{p}} L_{d} i_{d}+\omega_{\Psi_{p}} \Psi_{p}$,

$m=\frac{3}{2} p\left(\left(L_{d}-L_{q}\right) i_{d} i_{q}+\Psi_{p} i_{q}\right)$,

$\Theta \frac{d \omega}{d t}=m-m_{l}-F \omega$,

where $u_{d}$ is the $d$ component of the stator voltage, $R$ is the stator resistance, $i_{d}$ is the $d$ component of stator current, $L_{d}$ is the inductance in the $d$-direction, $L_{q}$ is the inductance in the $q$-direction, $i_{q}$ is the $q$ component of stator current, $u_{q}$ is the $q$ component of the stator voltage, $\Psi_{p}$ is the pole-flux vector's amplitude, $m$ is the machine's electromagnetic torque, $m_{l}$ is the load torque on the shaft, $\Theta$ is the 
rotor's moment of inertia, $F$ is the friction loss factor, $p$ is the number of pole pairs, $\omega$ is the shaft angular speed and $\omega_{\Psi_{p}}$ is the pole-flux vector's angular speed where $\omega_{\Psi_{p}}=p \omega$.

\subsection{High-Frequency Synchronous Injection}

In case of high-frequency synchronous injection, the test vectors are injected in the estimated $\hat{d}-\hat{q}$ frame shown in Fig. 2(a). This figure also explains the angle relations, which were used during the modeling process; based on Eq. (5), the angle displacement between the real and estimated coordinate systems is considered to be positive, if the estimated angle lags behind the real one.

$\alpha_{e}=\alpha_{\Psi_{p}}-\hat{\alpha}_{\Psi_{p}}$,

where $\alpha_{\Psi_{p}}$ is the pole flux vector's angle, $\hat{\alpha}_{\Psi_{p}}$ is its estimated value. In the following, the hat symbol ( ${ }^{\wedge}$ ) will denote the estimated values, $h$ subscripts will refer to high-frequency components. Fig. 2(b) illustrates the block diagram of the injection method, where the signals, which will be detailed, are indicated.

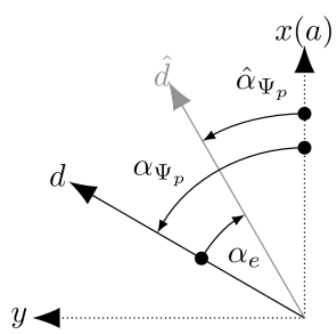

(a)

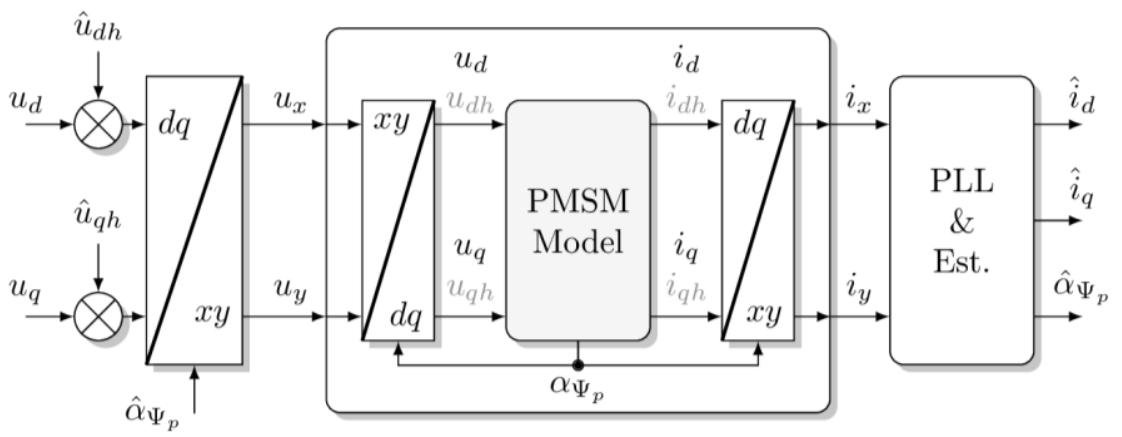

(b)

Figure 2

(a) Angle relation between the real $d-q$ and estimated $\hat{d}-\hat{q}$ coordinate systems (b) Block diagram of synchronous injection 
The injected voltages are not DC like quantities as they are used to be in the $d-q$ frame, but high-frequency sinusoidal signals. During the mathematical modeling, these time signals were handled using complex phasors, where the complex rotating vector was bound to the sine wave. According to this, the injected voltages are the following in both time and complex frequency domain,

$\left[\begin{array}{l}\hat{u}_{d h} \\ \hat{u}_{q h}\end{array}\right]=\left[\begin{array}{c}u_{h} \sin \left(\omega_{h} t\right) \\ -u_{h} \cos \left(\omega_{h} t\right)\end{array}\right]=\left[\begin{array}{c}u_{h} \\ -j u_{h}\end{array}\right]$,

where $\hat{u}_{d h}$ and $\hat{u}_{q h}$ are the injected voltages in the estimated $\hat{d}$-and $\hat{q}$-directions, $u_{h}$ is the amplitude of the injected voltages, $\omega_{h}=2 \pi f_{h}$, where $f_{h}$ is the injection frequency, $j$ is the imaginary unit.

With these definitions of the angle displacement and the injected voltages, the projections of the high-frequency signals on the real $d-q$ axes can be calculated using the rotation operator, as

$\left[\begin{array}{l}u_{d h} \\ u_{q h}\end{array}\right]=\overline{\bar{R}}\left(\alpha_{e}\right)\left[\begin{array}{l}\hat{u}_{d h} \\ \hat{u}_{q h}\end{array}\right]$,

where $\overline{\bar{R}}\left(\alpha_{e}\right)$ is the rotational operator and

$\overline{\bar{R}}\left(\alpha_{e}\right)=\left[\begin{array}{cc}\cos \left(\alpha_{e}\right) & \sin \left(\alpha_{e}\right) \\ -\sin \left(\alpha_{e}\right) & \cos \left(\alpha_{e}\right)\end{array}\right]$

and its inverse can be calculated as follows,

$\overline{\bar{R}}^{-1}\left(\alpha_{e}\right)=\left[\begin{array}{cc}\cos \left(\alpha_{e}\right) & -\sin \left(\alpha_{e}\right) \\ \sin \left(\alpha_{e}\right) & \cos \left(\alpha_{e}\right)\end{array}\right]$.

Equations (8)-(9) are not only valid for $\alpha_{e}$ and Eq. (7), but any given $\alpha$ angle. This method uses test voltages; therefore, the current response of the system could be calculated using Ohm's law as shown in Eq. (10).

$\left[\begin{array}{l}u_{d h} \\ u_{q h}\end{array}\right]=\overline{\bar{Z}}_{h}\left[\begin{array}{l}i_{d h} \\ i_{q h}\end{array}\right]=\overline{\bar{Z}}_{h} \overline{\bar{R}}\left(\alpha_{\Psi_{p}}\right)\left[\begin{array}{l}i_{x h} \\ i_{y h}\end{array}\right]$,

where $i_{d h}$ and $i_{q h}$ are the high-frequency currents in the $d-q$ frame, $i_{x h}$ and $i_{y h}$ are the current is the stationary frame. This equation contains $\overline{\bar{Z}}_{h}$, the highfrequency impedance matrix of the machine, which can be derived from Eqs. (1)(4) on the injection frequency as

$\overline{\bar{Z}}_{h}=\left[\begin{array}{cc}R+j \omega_{h} L_{d} & -\omega_{\Psi_{p}} L_{q} \\ \omega_{\Psi_{p}}\left(L_{d}+\frac{\Psi_{p}}{i_{d}}\right) & R+j \omega_{h} L_{q}\end{array}\right] \approx\left[\begin{array}{cc}R+j \omega_{h} L_{d} & 0 \\ 0 & R+j \omega_{h} L_{q}\end{array}\right]=$ $\left[\begin{array}{cc}\bar{Z}_{h 11} & 0 \\ 0 & \bar{Z}_{h 22}\end{array}\right]$.

Equation (11) shows that the impedance matrix's main diagonal contains the $d$ and $q$-direction impedances, which are only the function of the motor parameters and the injection frequency. On the other hand, the anti-diagonal elements are the 
function of the actual state of the system, since they depend on the common coordinate system's angular speed and the actual $d$-direction current. These elements can be neglected comparing to the other elements because the injection frequency expected to be much higher, than the highest possible $\omega_{\Psi_{p}}$, removing the nonlinearity from Eq. (10) [13]. The measurements can only be performed in the stationary coordinate system, therefore Eq. (10) combined with Eq. (7) are also needed to be organized to express $i_{x h}$ and $i_{y h}$, which results in the following,

$\left[\begin{array}{l}i_{x h} \\ i_{y h}\end{array}\right]=\overline{\bar{R}}^{-1}\left(\alpha_{\Psi_{p}}\right) \overline{\bar{Z}}^{-1}{ }_{h} \overline{\bar{R}}\left(\alpha_{e}\right)\left[\begin{array}{l}\hat{u}_{d h} \\ \hat{u}_{q h}\end{array}\right]$,

where the current components are

$$
\begin{aligned}
& i_{x h}=\left(\cos \left(\alpha_{e}\right) \cos \left(\alpha_{\Psi_{p}}\right) \frac{1}{\bar{Z}_{h 11}}+\sin \left(\alpha_{e}\right) \sin \left(\alpha_{\Psi_{p}}\right) \frac{1}{\bar{Z}_{h 22}}\right) \hat{u}_{d h} \\
& +\left(\sin \left(\alpha_{e}\right) \cos \left(\alpha_{\Psi_{p}}\right) \frac{1}{\bar{Z}_{h 11}}-\cos \left(\alpha_{e}\right) \sin \left(\alpha_{\Psi_{p}}\right) \frac{1}{\bar{Z}_{h 22}}\right) \hat{u}_{q h}, \\
& i_{y h}=\left(\cos \left(\alpha_{e}\right) \sin \left(\alpha_{\Psi_{p}}\right) \frac{1}{\bar{Z}_{h 11}}-\sin \left(\alpha_{e}\right) \cos \left(\alpha_{\Psi_{p}}\right) \frac{1}{\bar{Z}_{h 22}}\right) \hat{u}_{d h} \\
& +\left(\sin \left(\alpha_{e}\right) \sin \left(\alpha_{\Psi_{p}}\right) \frac{1}{\bar{Z}_{h 11}}-\cos \left(\alpha_{e}\right) \cos \left(\alpha_{\Psi_{p}}\right) \frac{1}{\bar{Z}_{h 22}}\right) \hat{u}_{q h} .
\end{aligned}
$$

\subsection{Signal Processing}

Equations (13)-(14) clearly show, that the high-frequency current response is in relation to the angle displacement between the real and estimated coordinate systems, so they can be used as an input for an angle estimator algorithm. In most cases, a Phase-Locked-Loop (PLL) structure is used, which block diagram is illustrated in Fig. 3.

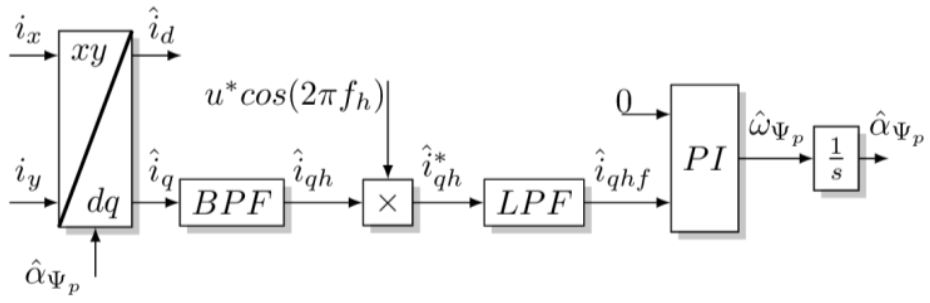

Figure 3

PLL structure used for angle estimation

In the first step of the estimation, the measurable stationary coordinate system currents are transformed into the estimated reference frame, as shown in Eq. (15). This equation combines the rotational operator and BPF block of Fig. 3, where $B P F$ is the abbreviation for band-pass-filter, which lets through the current components around the injection frequency region. 
$\left[\begin{array}{l}\hat{\imath}_{d h} \\ \hat{l}_{q h}\end{array}\right]=\overline{\bar{R}}\left(\hat{\alpha}_{\Psi_{p}}\right)\left[\begin{array}{l}i_{x h} \\ i_{y h}\end{array}\right]$

where

$$
\begin{aligned}
& \hat{\imath}_{d h}=\left(\cos ^{2}\left(\alpha_{e}\right) \frac{1}{\bar{Z}_{h 11}}+\sin ^{2}\left(\alpha_{e}\right) \frac{1}{\bar{Z}_{h 22}}\right) \hat{u}_{d h} \\
& +\sin \left(\alpha_{e}\right) \cos \left(\alpha_{e}\right)\left(\frac{1}{\bar{Z}_{h 11}}-\frac{1}{\bar{Z}_{h 22}}\right) \hat{u}_{q h}, \\
& \hat{\imath}_{q h}=\sin \left(\alpha_{e}\right) \cos \left(\alpha_{e}\right)\left(\frac{1}{\bar{Z}_{h 11}}-\frac{1}{\bar{Z}_{h 22}}\right) \hat{u}_{d h} \\
& +\left(\sin ^{2}\left(\alpha_{e}\right) \frac{1}{\bar{Z}_{h 11}}+\cos ^{2}\left(\alpha_{e}\right) \frac{1}{\bar{Z}_{h 22}}\right) \hat{u}_{q h} .
\end{aligned}
$$

Equation (17) contains both $\hat{u}_{d h}$ and $\hat{u}_{q h}$ which allows three possible solutions. In the first one, both $\hat{u}_{d h}$ and $\hat{u}_{q h}$ are used, as shown in Eq. (6). This approach is called synchronous rotating injection, for which authors in [14] proposed a demodulation algorithm. The other two solutions, which are simply called synchronous injection, use only one of the available voltages. Authors in [15] discussed the case, when the high-frequency voltage is applied in the estimated $\hat{q}$ axis, but in most of the cases only $\hat{u}_{d h}$ is injected, so Eq. (17) becomes as follows,

$\hat{\imath}_{q h}=\sin \left(\alpha_{e}\right) \cos \left(\alpha_{e}\right)\left(\frac{1}{\bar{z}_{h 11}}-\frac{1}{\bar{z}_{h 22}}\right) \hat{u}_{d h}$,

which is a complex phasor and its equivalent time signal is shown in Eq. (19).

$\hat{\imath}_{q h}(t)=u_{h} \sin \left(\alpha_{e}\right) \cos \left(\alpha_{e}\right)\left|\frac{1}{\overline{\bar{Z}}_{h 11}}-\frac{1}{\overline{\bar{Z}}_{h 22}}\right| \sin \left(\omega_{h} t+\operatorname{arc}\left(\frac{1}{\overline{\bar{Z}}_{h 11}}-\frac{1}{\overline{\bar{Z}}_{h 22}}\right)\right)$

This signal is fed into the phase detector, where it is multiplied with a cosine function having the same frequency as the injected voltage and amplitude $u^{*}$. The phase detector's output can be split into two components as shown in Eq. (20).

$$
\begin{aligned}
& \hat{\imath}_{q h}{ }^{*}(t)=\hat{l}_{q h}(t) u^{*} \cos \left(\omega_{h} t\right)= \\
& \frac{1}{2} u_{h} u^{*} \sin \left(\alpha_{e}\right) \cos \left(\alpha_{e}\right)\left|\frac{1}{\bar{Z}_{h 11}}-\frac{1}{\bar{Z}_{h 22}}\right| \sin \left(\operatorname{arc}\left(\frac{1}{\bar{Z}_{h 11}}-\frac{1}{\bar{Z}_{h 22}}\right)\right)+ \\
& \frac{1}{2} u_{h} u^{*} \sin \left(\alpha_{e}\right) \cos \left(\alpha_{e}\right)\left|\frac{1}{\overline{\bar{Z}}_{h 11}}-\frac{1}{\bar{Z}_{h 22}}\right| \sin \left(2 \omega_{h} t+\operatorname{arc}\left(\frac{1}{\bar{Z}_{h 11}}-\frac{1}{\bar{Z}_{h 22}}\right)\right) .
\end{aligned}
$$

One of these components is a DC-like quantity, whilst the other has twice frequency as the injection one. The latter can be filtered off using a Low-PassFilter, which is referred as $L P F$ in Fig. 3, resulting $\hat{\imath}_{q h f}(t)$, as shown in Eq. (21).

$$
\hat{\imath}_{q h f}(t) \approx \frac{1}{2} u_{h} u^{*} \sin \left(\alpha_{e}\right) \cos \left(\alpha_{e}\right)\left|\frac{1}{\overline{\bar{Z}}_{h 11}}-\frac{1}{\overline{\bar{Z}}_{h 22}}\right| \sin \left(\operatorname{arc}\left(\frac{1}{\overline{\bar{z}}_{h 11}}-\frac{1}{\overline{\bar{Z}}_{h 22}}\right)\right) \text {. }
$$


$\hat{\imath}_{q h f}(t)$ is fed into the PLL's PI controller as a feedback signal, and the controller's reference is set to zero. Observing Eq. (21) the feedback signal can be zero in four possible ways:

1. $u_{h}$ or $u^{*}$ equals zero,

2. $\left|\frac{1}{\bar{Z}_{h 11}}-\frac{1}{\bar{Z}_{h 22}}\right|$ becomes zero. In this case the machine is fully symmetrical in magnetic point of view, so no high-frequency signal injection methods can be applied, because Eqs. (13-17) will not contain any information from the angle error,

3. $\operatorname{arc}\left(\frac{1}{\bar{z}_{h 11}}-\frac{1}{\bar{z}_{h 22}}\right)$ becomes zero, which also means that the highfrequency impedances are equal,

4. $\sin \left(\alpha_{e}\right) \cos \left(\alpha_{e}\right)$ becomes zero, which means that the angle displacement between the real and estimated reference frames disappears.

This list clearly shows, that beside the trivial case when $u_{h}$ or $u^{*}$ is set zero, this method cannot deliver any angle information in case of machines, where the $d$ and $q$-direction high-frequency impedances are equal. Such machine could be a surface mounted permanent magnet synchronous machine, where $L_{d}$ and $L_{q}$ are the same. On the other hand, the zero setpoint of the controller is the result of the fourth point of this list; the angular displacement is zero between the real and estimated coordinate systems if the PI controller reaches its zero setpoint.

\subsection{Dynamic Model for the PLL Structure}

The tuning of PLL's controller requires a dynamic model, which can be created with the respect of the estimator structure. Equations (6)-(21) are valid in steady state, since phasors were involved in the calculations, but these results can be used to obtain the dynamic model. The proposed closed loop structure is illustrated in Fig. 4.

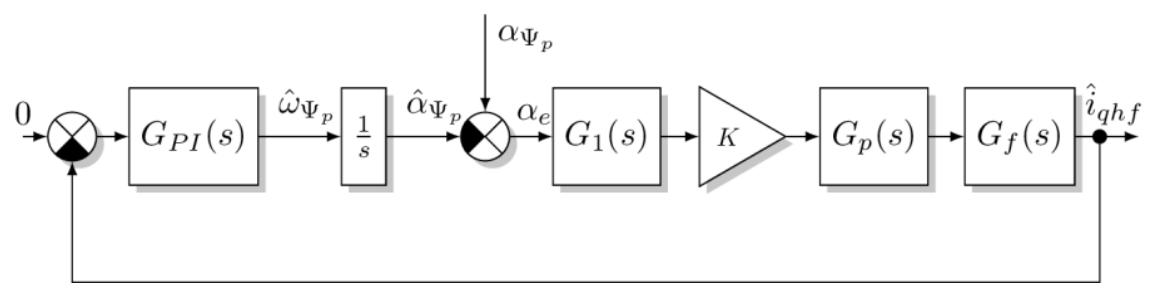

Figure 4

Dynamic model of the PLL

$G_{P I}(s)$ denotes a PI type controller, which was taken into account with the ideal form, so

$G_{P I}(s)=A_{p}\left(1+\frac{1}{s T_{i}}\right)$, 
where $A_{p}$ is the proportional gain, $T_{i}$ is the integral time. The controller's output, which is the estimated pole-flux vector angular speed, is fed into an integrator, resulting in the estimated value of the rotor flux vector's angle. The dynamic model's upcoming parts are can be constructed using the steady state current response in Eq. (21). According to this, the real and estimated angle's difference will be the input of $G_{1}(s)$, which is the operator domain transfer function of the $y=\sin (u) \cos (u)$ expression. The angle error is assumed relatively small during normal operation, so the transfer function $G_{1}(s)$ can be modeled with unity gain, therefore

$G_{1}(s)=1$.

$G_{p}(s)$ denotes the plant's transfer function, which is created using both $d$ - and $q$ direction R-L circuits, which is depicted in Fig. 5. In order to obtain the $s$ domain transfer function, the inductances are assumed to be non-energized in the $t=0$ step time, and the input voltage to be a sine wave step function, so

$u(t)=\sin \left(\omega_{h} t\right) \varepsilon(t)$,

where $\varepsilon(t)$ is the Heaviside step function. The current response can be calculated using test functions, where the steady state current is assumed to be as follows

$\mathrm{i}_{\text {stac }}(t)=\mathrm{i}_{\text {stac }} \sin \left(\omega_{h} t+\varphi\right)$,

where $\mathrm{i}_{\text {stac, } \mathrm{d}}=\left|\frac{1}{\mathrm{R}+\mathrm{j} \omega_{\mathrm{h}} \mathrm{L}_{\mathrm{d}}}\right|, \quad \varphi_{d}=\operatorname{arc}\left(\frac{1}{\mathrm{R}+\mathrm{j} \omega_{\mathrm{h}} \mathrm{L}_{\mathrm{d}}}\right)$ in case of the $d$-direction impedance, and $\mathrm{i}_{\text {stac, } \mathrm{q}}=\left|\frac{1}{\mathrm{R}+\mathrm{j} \omega_{\mathrm{h}} \mathrm{L}_{\mathrm{q}}}\right|, \varphi_{q}=\operatorname{arc}\left(\frac{1}{\mathrm{R}+\mathrm{j} \omega_{\mathrm{h}} \mathrm{L}_{\mathrm{q}}}\right)$ in case of the $q$-direction impedance.

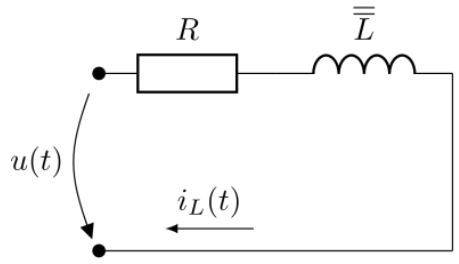

Figure 5

$d$ - and $q$-direction equivalent circuit

The first order system's response is sought as shown in Eq. (25).

$i_{L}(t)=\mathrm{Me}^{\lambda \mathrm{t}}+\mathrm{i}_{\mathrm{stac}}(t)$,

where $\lambda_{\mathrm{d}}=-\frac{\mathrm{R}}{\mathrm{L}_{\mathrm{d}}}, \lambda_{\mathrm{q}}=-\frac{\mathrm{R}}{\mathrm{L}_{\mathrm{q}}}$ for both directions, $M$ is a constant. With the respect of the boundary value problem, the current response time signal will be the following:

$i_{L}(-0)=i_{L}(+0)=\mathrm{M}+\mathrm{i}_{\text {stac }} \sin (\varphi)=0$, 
$M=-\mathrm{i}_{\text {stac }} \sin (\varphi)$,

$i_{L}(t)=\left(-\mathrm{i}_{\text {stac }} \sin (\varphi) \mathrm{e}^{\lambda t}+\mathrm{i}_{\text {stac }}(t)\right) \varepsilon(t)$.

According to Eq. (18) the current response fed into the phase detector is the difference of the $d$ - and $q$-direction responses, so

$$
\begin{aligned}
& \hat{\imath}_{q h}(t)=-\mathrm{i}_{\text {stac, } \mathrm{d}} \sin \left(\varphi_{d}\right) \mathrm{e}^{\lambda_{\mathrm{d}} \mathrm{t}}+\mathrm{i}_{\text {stac, } \mathrm{q}} \sin \left(\varphi_{q}\right) \mathrm{e}^{\lambda_{\mathrm{q}} \mathrm{t}} \\
& +\mathrm{i}_{\text {stac }, \mathrm{d}} \sin \left(\omega_{h} t+\varphi_{d}\right)-\mathrm{i}_{\text {stac, } \mathrm{q}} \sin \left(\omega_{h} t+\varphi_{q}\right)= \\
& -\mathrm{i}_{\text {stac }, \mathrm{d}} \sin \left(\varphi_{d}\right) \mathrm{e}^{\lambda_{\mathrm{d}} \mathrm{t}}+\mathrm{i}_{\text {stac }, \mathrm{q}} \sin \left(\varphi_{q}\right) \mathrm{e}^{\lambda_{\mathrm{q}} \mathrm{t}} \\
& +\mathrm{c} \sin \left(\omega_{h} t+\varphi_{d}+\varphi^{*}\right),
\end{aligned}
$$

where

$$
\begin{aligned}
c & =\sqrt{i_{\text {stac }, \mathrm{d}}{ }^{2}+\mathrm{i}_{\text {stac }, \mathrm{q}}{ }^{2}-2 \mathrm{i}_{\text {stac }, \mathrm{d}} \mathrm{i}_{\text {stac }, \mathrm{q}} \cos \left(\varphi_{q}-\varphi_{d}\right)}, \\
\varphi^{*} & =\tan ^{-1}\left(\frac{-\mathrm{i}_{\text {stac }, \mathrm{q}} \sin \left(\varphi_{q}-\varphi_{d}\right)}{\mathrm{i}_{\mathrm{stac}, \mathrm{d}}+\mathrm{i}_{\text {stac }, \mathrm{q}} \cos \left(\varphi_{q}-\varphi_{d}\right)}\right) .
\end{aligned}
$$

In the PLL structure we are interested in only the envelope of the current response because the PI controller tries to force it to zero. Based on Eq. (29)-(31) this can be described as follows,

$e\left(\hat{\imath}_{q h}(t)\right)=k\left(-\mathrm{i}_{\text {stac }, \mathrm{d}} \sin \left(\varphi_{d}\right) \mathrm{e}^{\lambda_{\mathrm{d}} \mathrm{t}}+\mathrm{i}_{\text {stac, } \mathrm{q}} \sin \left(\varphi_{q}\right) \mathrm{e}^{\lambda_{\mathrm{q}} \mathrm{t}}+\mathrm{c} \sin \left(\varphi_{d}+\varphi^{*}\right)\right),(32$

where $e()$ denotes the envelope function and which equation needs to fulfill the boundary value problem and needs to have the steady state amplitude c. This will result $k=\frac{1}{\sin \left(\varphi_{d}+\varphi^{*}\right)}$, so

$e\left(\hat{\imath}_{q h}(t)\right)=-\frac{\mathrm{i}_{\text {stac, }} \sin \left(\varphi_{d}\right)}{\sin \left(\varphi_{d}+\varphi^{*}\right)} \mathrm{e}^{\lambda_{d} \mathrm{t}}+\frac{\mathrm{i}_{\text {stac }, \mathrm{q}} \sin \left(\varphi_{q}\right)}{\sin \left(\varphi_{d}+\varphi^{*}\right)} \mathrm{e}^{\lambda_{\mathrm{q}} \mathrm{t}}+\mathrm{c}$.

Equation (33) is the step response of the system, so its transfer function can be calculated as $s \mathcal{L}\left(e\left(\hat{\imath}_{q h}(t)\right)\right)$, which results in

$G_{p}(s)=\frac{p_{1} s^{2}+p_{2} s+p_{3}}{\left(s-\lambda_{\mathrm{d}}\right)\left(s-\lambda_{\mathrm{q}}\right)}$

where

$$
\begin{aligned}
& p_{1}=-\frac{\mathrm{i}_{\mathrm{stac}, \mathrm{d} \sin \left(\varphi_{d}\right)}}{\sin \left(\varphi_{d}+\varphi^{*}\right)}+\frac{\mathrm{i}_{\mathrm{stac}, \mathrm{q}} \sin \left(\varphi_{q}\right)}{\sin \left(\varphi_{d}+\varphi^{*}\right)}+\mathrm{c}=0, \\
& p_{2}=\frac{\mathrm{i}_{\mathrm{stac}, \mathrm{d} \sin \left(\varphi_{d}\right)}}{\sin \left(\varphi_{d}+\varphi^{*}\right)} \lambda_{\mathrm{q}}-\frac{\mathrm{i}_{\mathrm{stac}, \mathrm{q}} \sin \left(\varphi_{q}\right)}{\sin \left(\varphi_{d}+\varphi^{*}\right)} \lambda_{\mathrm{d}}-\mathrm{c} \lambda_{\mathrm{d}}-\mathrm{c} \lambda_{\mathrm{q}}, \\
& p_{3}=\mathrm{c} \lambda_{\mathrm{d}} \lambda_{\mathrm{q}} .
\end{aligned}
$$


In the control structure shown in Fig. $4 K$ remained as a yet unknown gain. This gain can be defined based on the PLL structure, especially its phase detector part and Eq. (21). The $\left|\frac{1}{\bar{Z}_{h 11}}-\frac{1}{\bar{Z}_{h 22}}\right|$ part of this equation equals $c$, whilst $\sin \left(\alpha_{e}\right) \cos \left(\alpha_{e}\right)$ part of it was dealt with $G_{1}(s)$ above. The rest of this equation will be the $K$ constant, so

$K=\frac{1}{2} u_{h} u^{*} \sin \left(\operatorname{arc}\left(\frac{1}{\bar{z}_{h 11}}-\frac{1}{\bar{Z}_{h 22}}\right)\right)=\frac{1}{2} u_{h} u^{*} \sin \left(\varphi_{d}+\varphi^{*}\right)$.

$G_{f}(s)$ denotes the loop filter of the PLL, which is used to a be a simple low pass filter, so the open loop transfer function of the dynamic model can be modeled as shown in Eq. (39).

$G_{o}(s)=-K A_{p}\left(1+\frac{1}{s T_{i}}\right) \frac{1}{s} \frac{p_{1} s^{2}+p_{2} s+p_{3}}{\left(s-\lambda_{\mathrm{d}}\right)\left(s-\lambda_{\mathrm{q}}\right)} \frac{1}{s T_{f}+1}$,

where $G_{o}(s)$ is the open loop transfer function, $T_{f}$ is the low-pass filter's time constant.

\section{Simulation Results}

The simulations were carried out on a permanent magnet synchronous machine and its parameters are listed in Table 1.

Table 1

Permanent Magnet Synchronous Machine Parameters

\begin{tabular}{cc}
\hline Parameter & Value \\
\hline$P_{n}$ & $1.1 \mathrm{~kW}$ \\
$U_{n}$ & $400 \mathrm{~V}$ \\
$i_{n}$ & $2.53 \mathrm{~A}$ \\
$R$ & $6.2 \Omega$ \\
$L_{d}$ & $20.025 \mathrm{mH}$ \\
$L_{q}$ & $40.17 \mathrm{mH}$ \\
$\Psi_{p}$ & $0.305 \mathrm{Vs}$ \\
$p$ & 3 \\
$F$ & $0.0011 \mathrm{Nms}$ \\
$m_{n}$ & $3.5 \mathrm{Nm}$ \\
\hline
\end{tabular}

Fig. 6 illustrates the cascade PI controller loops, where the ref subscript refers to the reference values. The tuning of the cascade control loop's PI controllers, which are taken into account using Eq. (22), are tuned based on the predefined cut off frequency and the damping constant methodology [16]. 


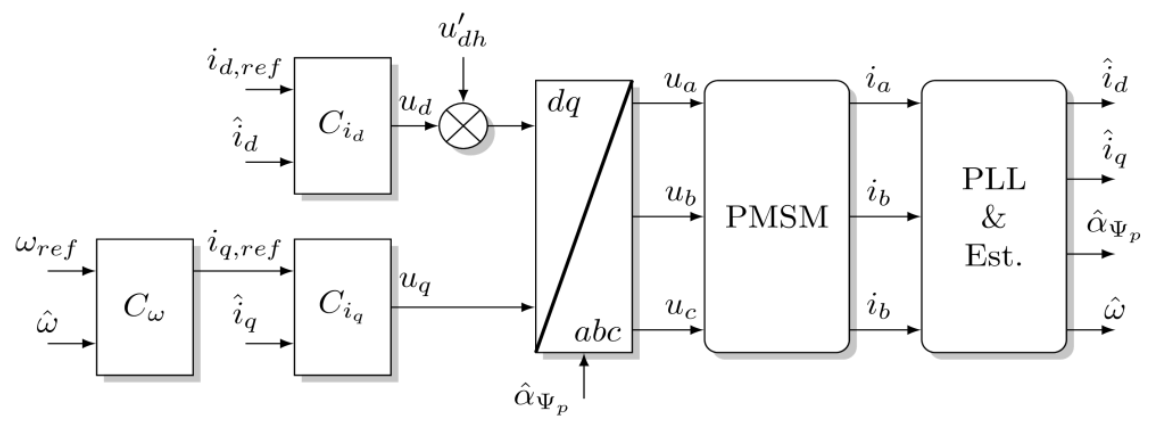

Figure 6

Cascade PI control loop

The overall performance of the whole cascade control loop depends on the estimator's PI controller, which can be tuned using the proposed dynamic model. In the first step of the simulation and tuning process, the injection frequency $f_{h}$ and the voltage amplitude $u_{h}$ must be defined with the respect of the highest possible $\omega_{\Psi_{p}}$. Figure 7 gives an overview of the effect of the selected frequency. The higher the injection frequency, the higher bandwidth can be achieved, but the signal amplitudes with which the PLL structure operates becomes smaller.
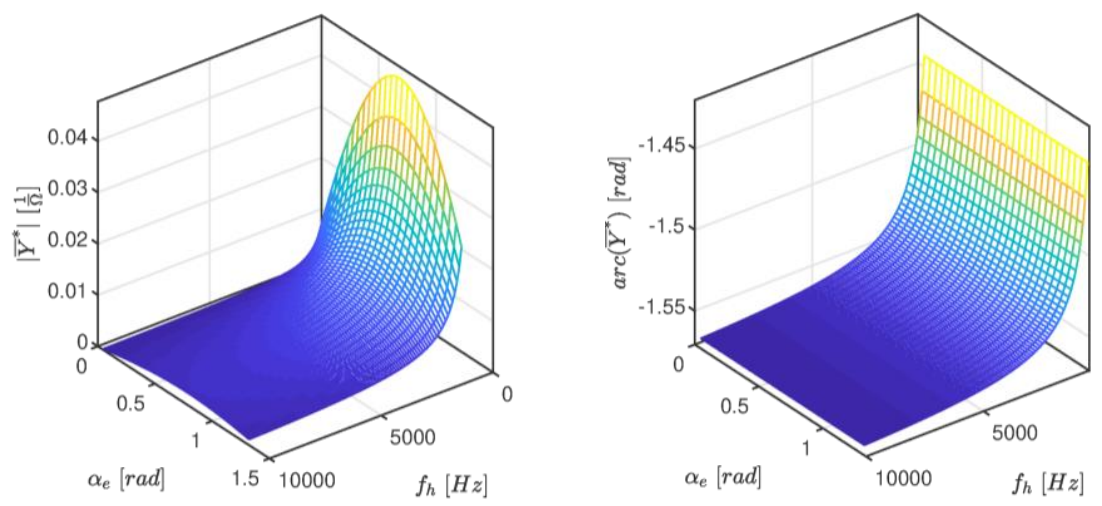

Figure 7

The amplitude and the phase of the response, where $\bar{Y}^{*}=\frac{1}{2} u_{h} u^{*} \sin \left(\alpha_{e}\right) \cos \left(\alpha_{e}\right)\left(\frac{1}{\bar{Z}_{h 11}}-\frac{1}{\bar{Z}_{h 22}}\right)$

The selected machine had 3 pole-pairs and nominal mechanical angular frequency of $50 \mathrm{~Hz}$, therefore the injection frequency was chosen to be $1500 \mathrm{~Hz}$, one decade higher than the expected highest possible $\omega_{\Psi_{p}}$ in normal operation. The high frequency voltage amplitude was $40 \mathrm{~V}$ and the phase detector's $u^{*}$ was set to 1 . During the simulations, no field weakening was examined. 
Based on Table 1, the process' Bode diagram including the $K$ gain is illustrated in Fig. 8. To obtain a stable control of the estimator, its PI controller's parameters were chosen to achieve 60 degrees phase margin [17] in the open loop Bode diagram, which is shown in Fig. 9.

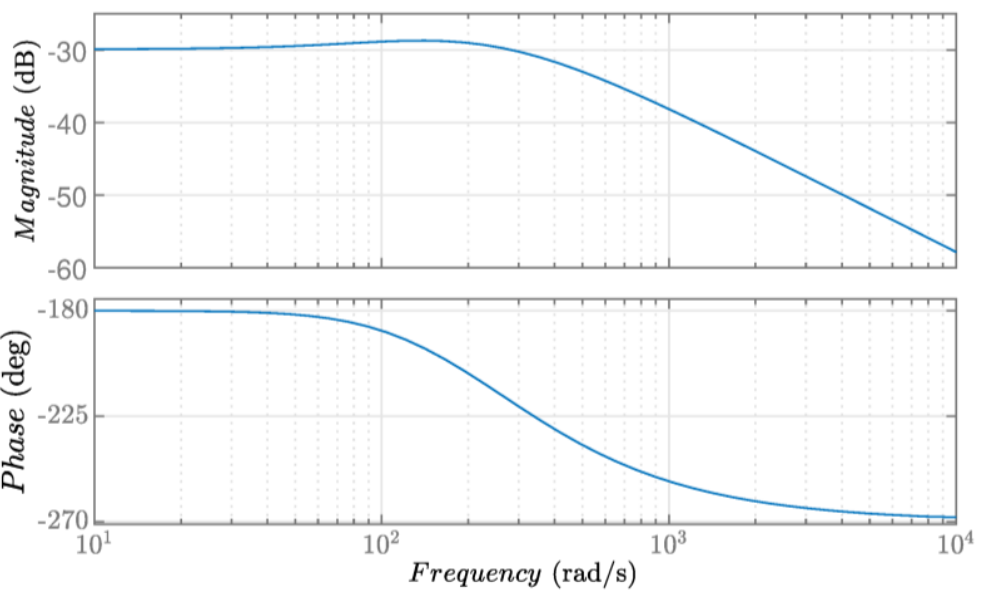

Figure 8

The process' Bode diagram
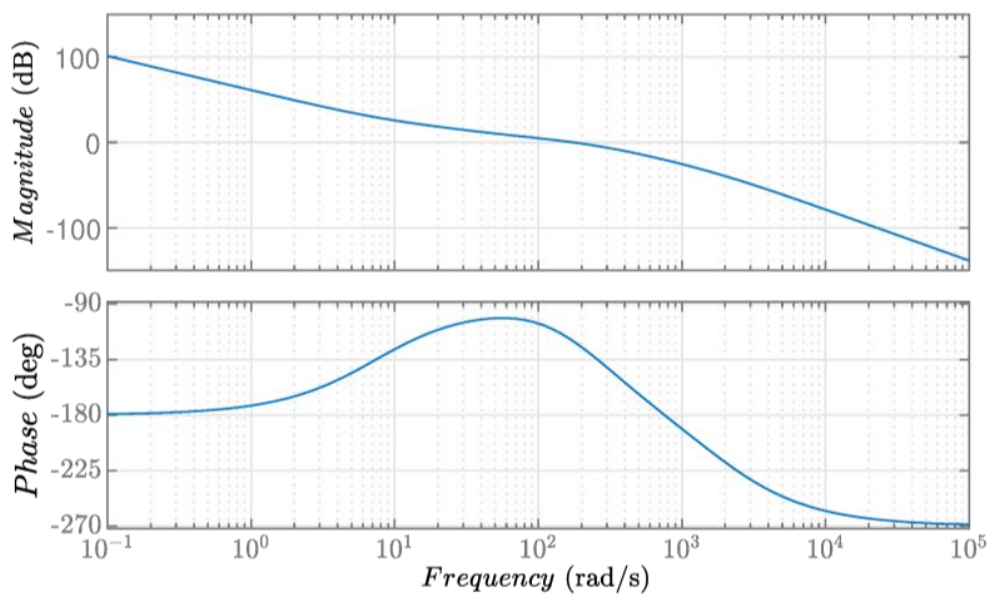

Figure 9

Open loop Bode diagram of the $G_{o}(s)$ PLL structure

The predefined phase margin belongs to $175 \mathrm{rad} / \mathrm{s}$ cut-off frequency in Fig. 9, so the speed control loop, which uses the estimator's angular velocity, must be tuned to be slower than this. Table 2 summarizes the PI controller and filter parameters, which were used in the cascade PI control loops and the estimator. 
Table 2

Controller parameters and filter time constants

\begin{tabular}{|c|c|c|}
\hline Parameter & Value & Description \\
\hline$A_{p, P L L}$ & 4853 & PLL's proportional gain \\
\hline$T_{i, P L L}$ & $136 \mathrm{~ms}$ & PLL's integral time \\
\hline$A_{p, d}$ & 11.49 & $d$-direction current controller proportional gain \\
\hline$T_{i, d}$ & $1.8 m s$ & $\begin{array}{l}d \text {-direction current controller } \\
\text { integral time }\end{array}$ \\
\hline$A_{p, q}$ & 23.92 & $q$-direction current controller proportional gain \\
\hline$T_{i, q}$ & $4.2 \mathrm{~ms}$ & $\begin{array}{l}q \text {-direction current controller } \\
\text { integral time }\end{array}$ \\
\hline$A_{p, \omega}$ & 0.287 & $\begin{array}{l}\text { angular speed controller } \\
\text { proportional gain }\end{array}$ \\
\hline$T_{i, \omega}$ & $36 m s$ & $\begin{array}{l}\text { angular speed controller } \\
\text { integral time }\end{array}$ \\
\hline$T_{f}$ & $0.53 \mathrm{~ms}$ & PLL's low-pass filter time constant \\
\hline
\end{tabular}

With these setting of the controllers and filters the following simulation results were obtained.
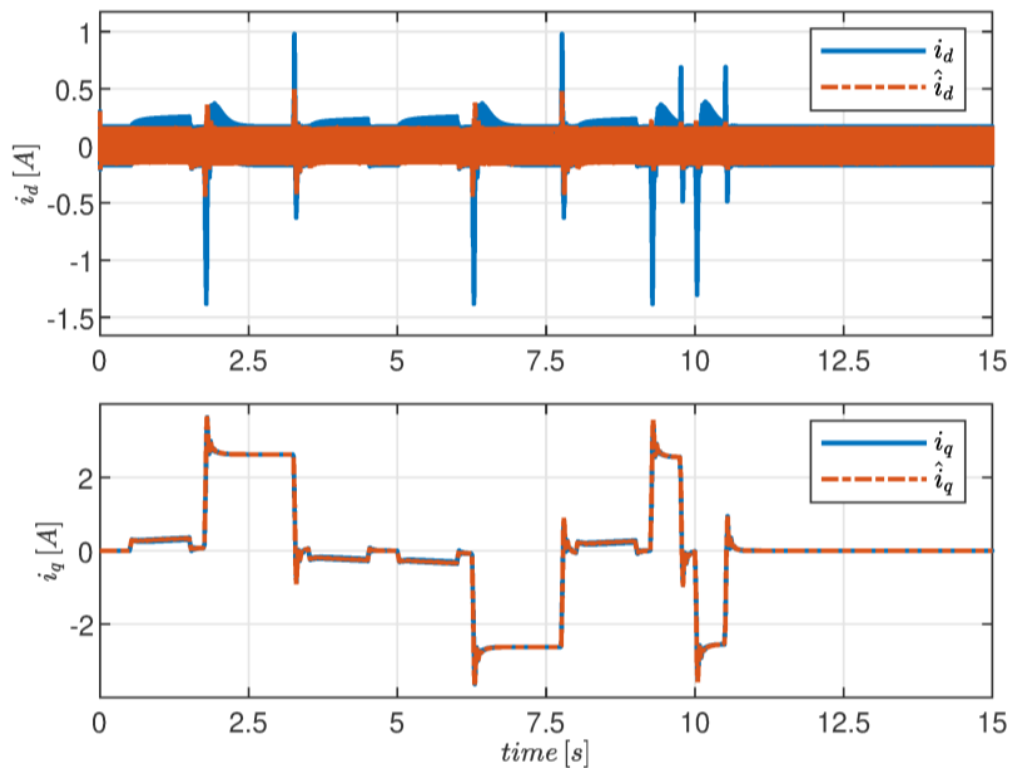

Figure 10

Simulated $d$ - and $q$-direction currents 

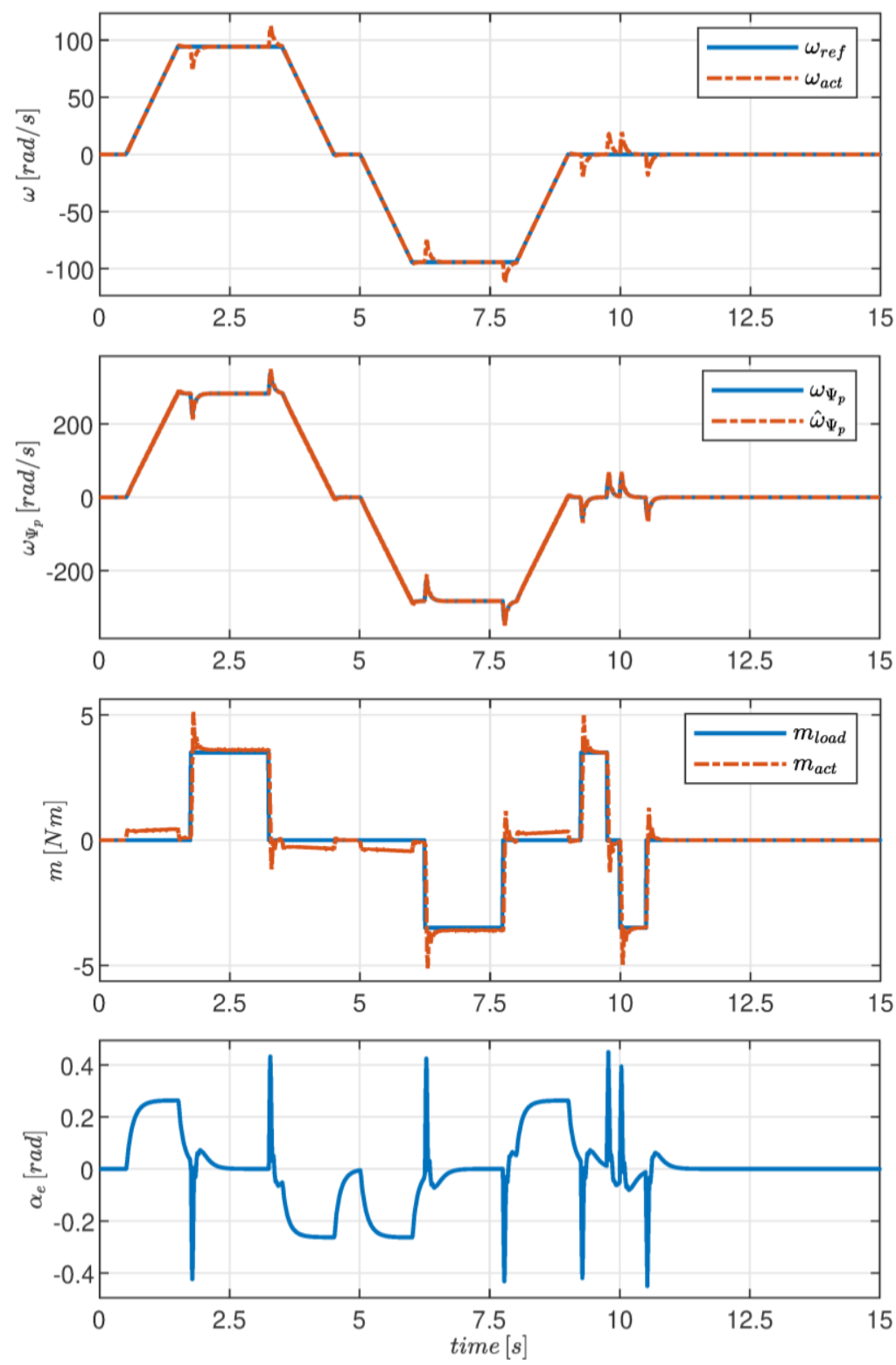

Figure 11

Simulated angular speed, rotor flux vector's angular speed, torque and angular displacement between the real and estimated coordinate systems

Figures 10 and 11 summarize the simulation results, including the actual, referred with act subscripts, and estimated signals of the machine. The speed-load profile was chosen to have positive and negative direction loads and angular speeds and 
zero-frequency cases with active load. The comparison of the field currents shows that the injection made its influence felt mainly in the flux branch of the system, which confirms that the injection voltage had only d-direction voltage component.

The implemented speed-sensorless algorithm was able to follow the speed and the torque requests, where the nominal torque was applied. The angle error also correlated with the control structure, since during dynamic changes, when the angular speed reference changes or torque is applied, the displacement between the real and estimated coordinate systems must occur, because it feeds the estimator's PI controller.

\section{Conclusions}

This paper presented the high-frequency synchronous voltage injection method on a permanent magnet synchronous machine. The mathematical model of this method was described, where it was shown that the method is unable to provide any angle information if the $d$ - and $q$-direction impedances are equal. The angle estimation depended on a PLL structure, for which a new dynamic model was proposed. With the help of this model, the PLL's PI type controller was tuned, which purpose is to force down its feedback signal to zero, in which condition the estimated and real coordinate systems' angle are the same. After tuning the estimator's controller, the widely used cascade control loop could be tuned and its performance could be analyzed.

The proposed dynamic model can be adapted to other types of machines, such as to squirrel-cage induction machines, synchronous reluctance machines. The ongoing research focuses on the application of high-frequency synchronous injection on different machines. High-frequency stationary injection is also investigated, where the Authors would like to report a comparison. Measurement results will be reported soon using a PWM IGBT inverter [18].

\section{References}

[1] M. Eskola and H. Tuusa: Comparison of MRAS and novel simple method for position estimation in PMSM drives, IEEE $34^{\text {th }}$ Annual Conference on Power Electronics Specialist, 2003, PESC '03, Acapulco, Mexico, 2003, pp. $550-555$ Vol. 2

[2] T. Orlowska-Kowalska and M. Dybkowski, Stator-current-based mrasestimator for a wide range speed-sensorless induction-motor drive, IEEE Transactions on industrial electronics, Vol. 57, No. 4, pp. 1296-1308, 2010

[3] H. M. Kojabadi and M. Ghribi, MRAS-based adaptive speed estimator in PMSM drives, $9^{\text {th }}$ IEEE International Workshop on Advanced Motion Control, 2006, Istanbul, 2006, pp. 569-572

[4] K. Wiedmann and A. Mertens, Self-sensing control of PM synchronous machines including online system identification based on a novel MRAS 
approach, $3^{\text {rd }}$ IEEE International Symposium on Sensorless Control for Electrical Drives (SLED 2012), Milwaukee, WI, 2012, pp. 1-8

[5] S. Bolognani, R. Oboe and M. Zigliotto, Sensorless full-digital PMSM drive with EKF estimation of speed and rotor position, IEEE Transactions on Industrial Electronics, Vol. 46, No. 1, pp. 184-191, Feb. 1999

[6] S. Bolognani, L. Tubiana and M. Zigliotto, Extended Kalman filter tuning in sensorless PMSM drives, IEEE Transactions on Industry Applications, Vol. 39, No. 6, pp. 1741-1747, Nov.-Dec. 2003

[7] M. Schroedl, Sensorless control of AC machines at low speed and standstill based on the "INFORM" method, IAS '96. Conference Record of the 1996 IEEE Industry Applications Conference Thirty-First IAS Annual Meeting, San Diego, CA, USA, 1996, pp. 270-277, Vol. 1

[8] J. M. Liu and Z. Q. Zhu, Sensorless Control Strategy by Square-Waveform High-Frequency Pulsating Signal Injection Into Stationary Reference Frame, IEEE Journal of Emerging and Selected Topics in Power Electronics, Vol. 2, No. 2, pp. 171-180, June 2014

[9] S. Kim, J. Im, E. Song and R. Kim, A New Rotor Position Estimation Method of IPMSM Using All-Pass Filter on High-Frequency Rotating Voltage Signal Injection, IEEE Transactions on Industrial Electronics, Vol. 63, No. 10, pp. 6499-6509, Oct. 2016

[10] D. Raca, P. Garcia, D. Reigosa, F. Briz and R. Lorenz, A comparative analysis of pulsating vs. rotating vector carrier signal injection-based sensorless control, 2008 Twenty-Third Annual IEEE Applied Power Electronics Conference and Exposition, Austin, TX, 2008, pp. 879-885

[11] Hyunbae Kim and R. D. Lorenz, Carrier signal injection based sensorless control methods for IPM synchronous machine drives, Conference Record of the 2004 IEEE Industry Applications Conference, 2004. $39^{\text {th }}$ IAS Annual Meeting., Seattle, WA, USA, 2004, pp. 977-984, Vol. 2

[12] Y. Yoon, S. Sul, S. Morimoto and K. Ide, High-Bandwidth Sensorless Algorithm for AC Machines Based on Square-Wave-Type Voltage Injection, IEEE Transactions on Industry Applications, Vol. 47, No. 3, pp. 1361-1370, May-June 2011

[13] Pozna, Claudiu, and Radu-Emil Precup. An Approach to the Design of Nonlinear State-Space Control Systems. Studies in Informatics and Control 27.1 (2018): 5-14

[14] C. Caruana, G. M. Asher, K. J. Bradley and M. Woolfson, Flux position estimation in cage induction machines using synchronous HF injection and Kalman filtering, IEEE Transactions on Industry Applications, Vol. 39, No. 5, pp. 1372-1378, Sept.-Oct. 2003 
[15] M. J. Corley and R. D. Lorenz, Rotor position and velocity estimation for a salient-pole permanent magnet synchronous machine at standstill and high speeds, IEEE Transactions on Industry Applications, Vol. 34, No. 4, pp. 784-789, July-Aug. 1998

[16] Wang, Liuping, et al. PID and predictive control of electrical drives and power converters using MATLAB/Simulink. John Wiley \& Sons, 2015

[17] Kiam Heong Ang, G. Chong and Yun Li, PID control system analysis, design, and technology, IEEE Transactions on Control Systems Technology, Vol. 13, No. 4, pp. 559-576, July 2005

[18] Vukosavic, Slobodan N. Digital control of electrical drives. Springer Science \& Business Media, 2007 